humains permettant un développement complet in vitro des stades hépatiques du parasite constituait

\section{Vers la vaccination anti-palustre}

L'enjeu de la mise au point d'une vaccination anti-palustre est considérable, comme en témoignent les quelques chiffres que nous rappelerons ici : l'affection menace le tiers de l'humanité, atteint 300 millions de personnes par an, et en tue deux à quatre millions.

Les crédits consacrés par les ÉtatsUnis aux recherches tendant à développer une vaccination efficace sont de l'ordre de 20 millions de dollars par an, et de nombreux autres pays dans le monde, notamment la France et l'Australie, sont également bien engagés dans cette voie de recherche. Il n'est pas possible ici de résumer dans leur diversité toutes les approches employées pour parvenir au résultat et nous n'évoquerons que les travaux portant sur la protéine majeure de surface des sporozoïtes (protéine circumsporozoïte). Les sporozoïtes constituent la forme infectante du parasite qui, accumulée dans les glandes salivaires du moustique anophèle, est injectée par l'insecte dans le courant sanguin.

Il est évidemment hors de question de développer à l'échelle de milliards d'individus une vaccination basée sur l'isolement de ces sporozoïtes qui doivent être préparés à partir des glandes salivaires de moustiques! Des anticorps dirigés contre les sporozoïtes ortt néanmoins été préparés à une petite échelle et se sont révélés protecteurs contre le paludisme de la souris. Ces anticorps reconnaissaient la protéine circumsporozoïte de plusieurs isolats de Plasmodium falciparum, l'agent de la forme la plus grave de paludisme. Cette dernière constatation achevait de faire de cette protéine un excellent candidat pour le développement du vaccin puisqu'il était démontré que des anticorps dirigés contre elle développaient une protection spécifique contre l'infection palustre et que l'absence de grandes variations antigéniques laissait espérer que le vaccin serait d'efficacité assez générale sur les différents isolats du parasite dans le monde. L'obtention d'une quantité suffisante de la protéine était dès lors l'affaire du génie génétique. En I984 deux groupes américains parvenaient à cloner le gène codant pour la protéine circumsporozoïte. L'un d'entre eux partait, pour obtenir les clones, d'une banque d'ADN compléinentaire des messagers isolés d'une préparation de sporozoïtes [I], l'autre utilisant une méthode différente qui fait appel à la construction d'une banque d'ADN génomique enrichie en gènes exprimés [2]. La séquence du gène révélait que la protéine circumsporozoïte était constituée de trente-sept répétitions du tétrapeptide asparagine-alanine-asparagine-proline et de quatre répétitions du tétrapeptide asparagine-valine-acide aspartique-proline $[\mathrm{I}, 2]$. L'anticorps monoclonal cité plus haut reconnaissait des peptides synthétiques identiques à la séquence répétée [2]. La première équipe avait, en 1983 , réussi à cloner le gène équivalent de Plasmodium knomlesi [3], responsable du paludisme du singe, et avait démontré que sa structure comportait une répétition d'une séquence codant pour un dodecapeptide. Un anticorps développé contre ce peptide synthétique se liait aux sporozoïtes et abolissait leur infectivité.

Deux voies s'ouvraient donc pour la préparation d'un vaccin : soit l'utilisation de peptides synthétiques, soit la production par génie génétique de la protéine circumsporozoïte dans sa totalité.

Le problème des essais préliminaires d'anticorps anti-palustres chez l'homme se pose avec acuité pour d'évidentes raisons éthiques. C'est dire que le développement par Dominique Mazier, du laboratöire du Pr. Gentilini à Paris, d'un système de culture d'hépatocytes une avancée importante [4]. La méthode originale de culture de cellules hépatiques qui, en présence d'une autre population cellulaire dérivée du foie, gardent in vitro l'essentiel de leur potentiel de différenciation pendant plusieurs mois, a été développée par Christiane Guillouzo à Rennes, dans le laboratoire $\mathrm{du}$ Professeur Bourrel. Utilisant donc ce système, Dominique Mazier et al. viennent de démontrer que les anticorps dirigés soit contre la protéine circumsporozoïte obtenue par génie génétique, soit contre des peptides synthétiques, s'opposent efficacement à l'infection des hépatocytes humains par des sporozoïtes de Plasmodium falciparum. L'action se fait à trois niveaux : l'attachement des sporozoïtes à la membrane cellulaire, leur entrée dans la cellule et leur développement intracellulaire [5].

Quoiqu'encourageants, ces résultats ne signifient malheureusement pas que la vaccination anti-palustre soit pour demain. D'une part rien n'est encore connu de l'antigénicité chez l'homme de la protéine clonée et des peptides synthétiques. D'autre part l'immunité anti-sporozoïte ne sera probablement pas totale et laissera échapper quelques parasites qui seront à l'origine d'accès palustres. Il n'empêche que si la gravité des accès pernicieux et de toutes les formes les plus graves est bien en rapport avec l'intensité de l'infestation, la vaccination et le développement d'une immunité antisporozoïte devraient au moins réduire considérablement la fréquence des formes mortelles.

A. $\mathbf{K}$

I. Enea V, Ellis J, Zavala F, et al. DNA cloning of Plasmodium falciparum circumsporozoïte gene. Aminoacid sequence of repetitive epitope. Science 1984; 225: 628-9.

2. Dame JB, Williams JL, McCutchan IF, et al. Structure of the gene encoding the immunodominant surface antigen on the sporozoite of the human malaria parasite Plasmodium falciparum. Science 1986; 225: 593-9.

3. Godson GN, Ellis J, Svec P, et al. Identification and chemical synthesis of a tandemly repeated immunogenic region of Plasmodium 
knowlesi circumsporozoïte protein. Nature 1983; 305: 29-33.

4. Mazier D, Beaudoin RL, Mellouk S, et al. Complete development of hepatic stages of Plasmodium falciparum in vitro. Science 1985;
227: 440-2.

5. Mazier D, Mellouk S, Beaudoin RL, et al. Effect of antibodies to recombinant and synthetic peptides on $P$. falciparum sporozoïtes in vitro Science 1986; 231: I56-9.

\section{Mais à quoi sert donc l'ubiquitine?}

L'ubiquitine est une protéine de 76 aminoacides (poids moléculaire : 8 500), présente dans toutes les cellules de pratiquement toutes les espèces eucaryotiques. Sa structure est extraordinairement conservée dans l'évolution: les protéines de certains amphibiens, de drosophile et d'homme sont identiques [I].

Son rôle le mieux connu est son intervention dans la dégradation protéolytique des protéines altérées ou à courte durée de vie (ce qui est le cas de la plupart des facteurs protéiques jouant un rôle de régulation). L'ubiquitine forme une liaison peptidique entre son radical $\mathrm{COOH}$ terminal et un radical $\varepsilon-\mathrm{NH}_{2}$ d'un résidu lysine de la protéine à dégrader.

Une telle structure "branchée " existe également au niveau de l'histone $2 \mathrm{~A}$ dont la liaison avec l'ubiquitine n'interviendrait pas ici dans la dégradation mais plutôt dans la régulation fonctionnelle.

Varschavsky et ses collègues (MIT, Cambridge, Mas, USA) ont en effet noté que l'histone $2 \mathrm{~A}$ " ubiquitinée " était préférentiellement localisé dans des régions où les gènes sont activement transcrits; l'hypothèse peut donc être faite que la fixation d'une molécule d'ubiquitine sur l'histone $2 \mathrm{~A}$ modifie la chromatine, provoquant peut-être un relâchement indispensable à l'expression du gène $[\mathrm{I}]$.

Il existe plusieurs gènes codant pour cette protéine; ils sont transcrits en des messagers qui codent pour des pré-protéines contenant plusieurs motifs ubiquitine, pré-protéines qui doivent donc subir une maturation protéolytique pour libérer les molécules isolées d'ubiquitine. Une lignée cellulaire incapable de catalyser la fixation de l'ubiquitine sur d'autres protéines, probablement par un déficit de l'activité enzymatique responsable de cette réaction, a été décrite $[\mathrm{I}]$. Ces cellules meurent quand la température est augmentée de 32 à $39^{\circ} \mathrm{C}$, ce qui renforce l'idée que ce système est notamment impliqué dans les défenses contre le choc thermique [2]. Le rôle de l'ubiquitine pourrait être ici de dégrader les protéines altérées par l'élévation de température, et peutêtre aussi de permettre la régulation de l'expression d'autres gènes dont les produits jouent aussi un rôle dans les phénomènes de défense contre le choc thermique [2].

De récents travaux du laboratoire de I.L. Weissman (Stanford, Ca, USA) viennent, de façon absolument inattendue, d'attirer l'attention sur d'autres fonctions de l'ubiquitine. Cette équipe étudie le récepteur lymphocytaire qui reconnaît un motif situé sur les veinules post-capillaires des organes lymphoïdes et est ainsi responsable de la captation par ces organes des lymphocytes circulants. A l'aide d'un anticorps monoclonal dirigé contre le récepteur (dit de "domiciliation "), les chercheurs du laboratoire de Weissman criblèrent une banque d'expression d'ADN complémentaire et isolèrent ainsi trois clones d'ADNc dont la séquence révèla qu'ils codaient tous pour l'ubiquitine [3]. L'isolement du récepteur montra qu'il. était composé d'une protéine dont les sous-unités avaient un poids moléculaire de 90000 et deux extrêmités N-terminales, l'une d'entre elles correspondant à une molécule d'ubiquitine "branchée " sur le récepteur [4]. C'est contre un épitope antigénique de cette molécule d'ubiquitine qu'était dirigé l'anticorps monoclonal utilisé pour cribler la banque d'ADNc.

Ainsi l'ubiquitine est-elle non seulement présente dans toutes les cellules, mais aussi dans leur différents compartiments : le noyau, le cytoplasme... et la face externe de la membrane. Son "ubiquité " pourrait ainsi être à la fois cellulaire, d'espèce, et fonctionnelle.

A. $\mathbf{K}$.

I. Finley D, Varschavsky A. The ubiquitin system: functions and mechanisms. Trends Biochem Sci 1985; 10, 343-9.

2. Munro S, Pelham $H$. What turns on heat shock genes? Nature 1985; 317: 477-8.

3. St John T, Gallatin MW, Spiegelman M. Expression cloning of a lymphocyte homing receptor $\mathrm{cDNA}$ : ubiquitin is the reactive species. Science 1986; 231: 845-50.

4. Spiegelman M, Bond MN, Gallatin MW. Cell surface molecule associated with lymphocyte homing is a ubiquitinated branched-chain glycoprotein. Science 1986; 231: 823-9.

\section{La mouche au secours des embryologistes}

\section{Les "homéoboxes" des mammifères sont situés dans des gènes exprimés au cours du développement.}

Les boîtes homéotiques ou "homéoboxes" sont ces séquences codantes de $\mathrm{I} 80$ paires de bases (pb) qui se retrouvent dans la drosophile au niveau des gènes homéotiques (c'està-dire de gènes impliqués dans la segmentation de l'animal [I]). 